\title{
Open system geometric phase based on system-reservoir joint state evolution
}

\author{
Shi-Biao Zheng* \\ Department of Physics, Fuzhou University, Fuzhou 350116, China
}

(Dated: January 13, 2021)

\begin{abstract}
The geometric phase is of fundamental interest and plays an important role in quantum information processing. However, the definition and calculation of this phase for open systems remains a problem due to the lack of agreement on generalizations of the parallel transport condition to mixed state nonunity evolutions. Here we tackle this problem by associating the open system geometric phase with the parallel transport of the joint system-reservoir state. Our approach not only provides a way around the nonunitary evolution obstacle, but also sheds light on the relation between the geometric phase and the system-reservoir entanglement, which has not been investigated. Based on this approach, we calculate the geometric phase of different quantum systems subject to energy decay, showing that it is robust against decoherence, which is in distinct contrast with previous results.

PACS numbers: 03.65.Vf, 03.65.Yz, 03.65.Ud
\end{abstract}

When the parameters of the Hamiltonian of a quantum system initially in an eigenstate of the Hamiltonian is changed in a cyclic and adiabatic fashion, it will return to the initial state but acquires a geometric phase in addition to the dynamical one $[1,2]$. This effect, known as the Berry phase, has attracted interest from a variety of fields [3] and been generalized in various ways. Aharonov and Anandan removed the adiabatic condition and showed that the geometric phase depends upon the geometry of the path traversed by the system in the projected Hilbert space [4]. Based on the work of Pancharatnam on the interference of polarized light [5], the geometric phase associated with a noncyclic evolution was discovered [6]. The generalization to mixed quantum states was first introduced by Uhlmann [7] and then developed by Sjoqvist et al., who defined the mixed state geometric phase due to unitary evolution based on interferometry [8]. In addition to fundamental interest, geometric phases have practical applications, among which the implementation of geometric quantum computation is a typical example. Quantum gates based on geometric phase may have intrinsic resistance to external noise, offering potential advantages as compared with dynamical ones [9-16].

One of the main obstacles for coherent control of quantum systems and implementation of quantum computation is decoherence due to the presence of noises, including fluctuations in the control parameters and unavoidable interaction with the environment. This leads to many theoretical and experimental studies of geometric phases under classical [17-24] and quantum noises [25-35]. For the latter case, one should deal with the geometric phase in an open system, whose definition is still an open problem though approaches have been developed for this purpose [25-28]. For different approaches the parallel transport conditions used to define mixed state geometric phases are not consistent $[26,36]$. In Ref. [27], Carollo et al. defined and calculated the geometric phase of a quantum system subject to docoherence through the quantum jump method. It was shown that the geometric phase is completely insensitive to dephasing but it is affected by the spontaneous decay. In this framework, Carollo et al. investigated the behavior of the geometric phase of a spin-1/2 particle interacting with a decohering quantum field, showing that to the first correction this phase is insensitive to decoherence for adiabatic evolution but is affected by decoherence for nonadiabatic evolution [28]. This approach avoids the problem of finding the parallel transport condition for mixed states, but it requires continuous observation of the reservoir state, which would interrupt the joint system-reservoir state evolution, destroying the entanglement between the system and environment. Furthermore, it is experimentally demanding to accurately detect whether a quantum jump has occurred or not. Another problem is that for any evolution trajectory involving one or more energy decay jumps the geometric phase cannot be well defined since in this case the final and initial states become orthogonal.

The aim of the present paper is to overcome these problems and to present a new method to define and calculate the geometric phase of quantum systems coupled to reservoirs. Our method, based on the evolution of the system-reservoir joint state, requires neither observation of quantum jumps nor tracing over the degrees of freedom of the reservoir. Since the reservoir state is included, the total system undergoes a unitary evolution and the parallel transport condition is well defined. Within this framework, the geometric phase is unique and depends upon the geometry of the path traversed by the joint system-environment state in the total Hilbert space. We show that this phase can be calculated without knowledge of the state components with one or more excitations having leaked to the reservoir. Based on

*Electronic address: sbzheng11@163.com 
this approach, we investigate the behavior of the geometric phase of different open systems, showing that this phase is robust against spontaneous decay. We also present a geometric explanation to this effect in view of the correlated system-environment state evolution. This interesting result is contrary to the previous study [26-28], which showed that the geometric phase for nonadiabatic evolution has no resistance to system decay. The reason for this difference is that in the previous works the system-environment entanglement is lost either by measuring the system decay or by tracing over the environmental degrees of freedom. We further show that for the Jaynes-Cummings (JC) model, the fundamental model describing the matter-light interaction at the quantum mechanical level, the open system geometric phase calculated through our approach is the only one that can be directly measured in experiment.

We consider a quantum system, whose Hamiltonian is given by $H_{s}$. Suppose that it is coupled to an $N$-mode reservoir. The total Hamiltonian is given by

$$
H_{s r}=H_{s}+H_{r}+H_{i}
$$

where $H_{r}=\hbar \sum_{k=1}^{N} \omega_{k} b_{k}^{\dagger} b_{k}$ is the reservoir Hamiltonian, $H_{i}=\hbar \sum_{k=1}^{N} g_{k}\left(b_{k}^{\dagger} o+b_{k} o^{\dagger}\right)$ describes the system-reservoir interaction, and $b_{k}^{\dagger}$ and $b_{k}$ are the creation and annihilation operators for the $k$ th reservoir mode with the frequency $\omega_{k}$, which is coupled to the system with the strength $g_{k}$. Here $o\left(o^{\dagger}\right)$ is the linear combination of the relevant lowering (raising) operators for the energy levels of the quantum system. Assume that both the system and reservoir are initially in pure states so that their evolution follows a definite trajectory $\left|\psi^{s r}(t)\right\rangle$ in the total Hilbert space of joint system-reservoir state under the total Hamiltonian $H_{s r}$. For nonvanishing system-reservoir entanglement, the total geometric phase acquired by the joint state evolution is inseparable. To give the parallel transport condition associated with this phase, we define the new state

$$
\left|\Phi^{s r}(t)\right\rangle=e^{-i \phi_{d}}\left|\psi^{s r}(t)\right\rangle
$$

where $\phi_{d}=-\int_{0}^{t}\left\langle\psi^{s r}\left(t^{\prime}\right)\left|H_{s r}\right| \psi^{s r}\left(t^{\prime}\right)\right\rangle d t^{\prime}$ is the dynamical phase for the total system. With the dynamical phase being removed, $\left|\Phi^{s r}(t)\right\rangle$ satisfies the parallel transport condition

$$
\left\langle\Phi^{s r}(t)\left|\frac{d}{d t}\right| \Phi^{s r}(t)\right\rangle=0
$$

After a time $T$ the geometric phase of the total system associated with this parallel transport is

$$
\begin{aligned}
\beta & =\arg \left\langle\Phi^{s r}(0) \mid \Phi^{s r}(T)\right\rangle \\
& =\phi-\phi_{d},
\end{aligned}
$$

where $\phi=\arg \left\langle\psi^{s r}(0) \mid \psi^{s r}(T)\right\rangle$ is the total phase difference between the initial and final states $\left|\psi^{s r}(0)\right\rangle$ and $\left|\psi^{s r}(T)\right\rangle$. The geometric phase defined in this way, acting as a memory of the joint motion of the system and environment, depends purely upon the geometry of the trajectory followed by the joint system-reservoir state in the total Hilbert space.

Suppose the system is initially in the state $\left|\psi_{0}^{s}(0)\right\rangle$ and the reservoir initially in the vacuum state $|\overline{0}\rangle=\prod_{k=1}^{N}\left|0_{k}\right\rangle$. The evolution of the total system is given by

$$
\left|\psi^{s r}(t)\right\rangle=\left|\psi_{0}^{s}(t)\right\rangle|\overline{0}\rangle+\left|\psi_{1}^{s r}(t)\right\rangle
$$

where $\left|\psi_{1}^{s r}(t)\right\rangle$ is the combined system-reservoir state associated with one or more excitations being transferred to the reservoir so that it is orthogonal to $\left|\psi_{0}^{s}(t)\right\rangle|\overline{0}\rangle$, which corresponds to no excitation transfer. The evolution of $\left|\psi_{0}^{s}(t)\right\rangle$ is governed by the conditional Hamiltonian $H_{s, c}=H_{s}-i \hbar \frac{\gamma}{2} o^{\dagger} o$, where $\gamma$ is the system energy decaying rate. Here $\left|\psi_{0}^{s}(t)\right\rangle$ and $\left|\psi_{1}^{s r}(t)\right\rangle$ are the unnormalized states, with $\left\langle\left.\psi_{0}^{s}(t)\right|_{0} ^{s}(t)\right\rangle$ being the probability with no excitation decaying to the reservoir and $\left\langle\psi_{1}^{s r}(t) \mid \psi_{1}^{s r}(t)\right\rangle$ the probability with one or more excitations in the reservoir modes.

When the system Hamiltonian is time independent, the total Hamiltonian is a conserved quantity and

$$
\phi_{d}=-\left\langle\psi^{s r}(0)\left|H_{s r}\right| \psi^{s r}(0)\right\rangle T \text {. }
$$

For the initially vacuum reservoir, we have $\left\langle\psi^{s r}(0)\left|H_{r}\right| \psi^{s r}(0)\right\rangle=\left\langle\psi^{s r}(0)\left|H_{i}\right| \psi^{s r}(0)\right\rangle=0$ and hence $\phi_{d}=$ $-\left\langle\psi^{s r}(0)\left|H_{s}\right| \psi^{s r}(0)\right\rangle T$. Since $\left\langle\psi^{s r}(0) \mid \psi_{1}^{s r}(t)\right\rangle=0$, the total phase difference between the initial and final states reducs to $\phi=\arg \left\langle\psi_{0}^{s}(0) \mid \psi_{0}^{s}(T)\right\rangle$. This implies that the geometric phase is indepenent of expression of $\left|\psi_{1}^{s r}(t)\right\rangle$, which 
makes the calculation of the geometric phase very simple. It should be noted that this geometric phase can be directly measured in the system interference experiment although it is obtained by the system-reservoir joint state [37]. The total phase obtained by the present approach is the same as that calculated through the quantum jump method for the no-jump trajectory $[27,28]$. The dynamical phase calculated through the quantum jump method is given by

$$
\phi_{d}^{\prime}=-\int_{0}^{T} \frac{\left\langle\psi_{0}^{s}(t)\left|H_{s}\right| \psi_{0}^{s}(t)\right\rangle}{\left\langle\psi_{0}^{s}(t) \mid \psi_{0}^{s}(t)\right\rangle} d t
$$

Since the evolution $\left|\psi_{0}^{s}(t)\right\rangle$ is governed by the non-Hermitian Hamiltonian $H_{s, c}$, the system Hamiltonian $H_{s}$ is not a conserved quantity for no-jump trajectory $\left|\psi_{0}^{s}(t)\right\rangle$ and hence $\phi_{d}^{\prime} \neq \phi_{d}$. This implies that the geometric phases obtained through these two approaches are different. This difference has a simple explanation. In the present method the geometric phase is associated with the real joint state evolution trajectory in the total Hilbert space, while in the quantum jump method it depends upon the projection of evolution trajectory to the subspace with no excitation in the reservoir modes. In other words, observation of the environment destroys the system-environment entanglement, changing the state trajectory of the total system and, therefore, the related geometric phase. It should be noted that for the no-jump trajectory the reservoir does not evolve and acquires no geometric phase, and therefore the geometric phase acquired by the system is also that acquired by the total system including the reservoir.

Let us analyze the geometric phases of typical physical systems based on this approach. We first consider the evolution of a two-level atom in a reservoir. This atom dispersively interacts with a classical electromagnetic field, which does not induce the atomic transition, but shifts the atomic energy levels. We here works in the framework rotating at the transition frequency $\omega_{a}$ of the atom without energy shift. Then the system Hamiltonian is given by $H_{s}=\frac{1}{2} \hbar B(|e\rangle\langle e|-| g\rangle\langle g|)$, where $|e\rangle$ and $|g\rangle$ are the upper and lower levels of the atom and $B$ characterizes the relative energy level shift caused by the off-resonant classical field. The system-reservoir Hamiltonian has the form of Eq. (1), with the frequency $\omega_{k}$ of the $k$ th reservoir mode being replaced by the detuning $\delta_{k}=\omega_{k}-\omega_{a}$. Suppose the system is initially in the state $\left.\left|\psi_{0}^{s}(0)\right\rangle=\cos (\theta / 2)|e\rangle+\sin (\theta / 2)|g\rangle\right)$. When the reservoir modes are closely spaced in frequency, the system-reservoir state evolution is given by Eq. (5), where [38]

$$
\begin{aligned}
\left|\psi_{0}^{s}(t)\right\rangle & =e^{-\gamma t / 2-i \varphi / 2} \cos (\theta / 2)|e\rangle+e^{i \varphi / 2} \sin (\theta / 2)|g\rangle, \\
\left|\psi_{1}^{s r}(t)\right\rangle & =e^{-i \varphi / 2 \sqrt{1-e^{-\gamma t}} \cos (\theta / 2)|g\rangle|\overline{1}\rangle}
\end{aligned}
$$

$\varphi=B t,|\overline{1}\rangle=\sum_{k=1}^{N} \lambda_{k}\left|0_{1} \ldots 0_{k-1} 1_{k} 0_{k+1} \ldots 0_{N}\right\rangle$ is the normalized collective state for the reservoir modes with one excitation, and $\gamma$ is the decaying rate of the atom due to interaction with the reservoir. Since the total Hamiltonian is conserved during the evolution, we have $\langle\overline{1} \mid \dot{\overline{1}}\rangle=0$. The total phase difference between the initial and final states is $\phi=\arg \left[e^{-\gamma T / 2-i B T / 2} \cos ^{2}(\theta / 2)+e^{i B T / 2} \sin ^{2}(\theta / 2)\right]$, while dynamical phase accumulated by the overall system is $\phi_{d}=-\frac{1}{2} B T \cos \theta$. Remarkably, for $B T=2 \pi$ (cyclic evolution in the absence of decoherence) the geometric phase of the total system is $\beta=-\pi(1-\cos \theta)$, which is completely unaffected by decoherence. For $\theta=\pi / 2$, the dynamical phase accumulated by the overall system is zero and the phase $\phi$ is of purely geometric origin.

To interpret this result, we rewrite the state vector of the whole system as

$$
\left|\psi^{s r}(t)\right\rangle=e^{-i \varphi / 2}\left[\cos (\theta / 2)|\bar{e}\rangle+e^{i \varphi} \sin (\theta / 2)|\bar{g}\rangle\right]
$$

where $|\bar{e}\rangle=e^{-\gamma t / 2}|e\rangle|\overline{0}\rangle+\sqrt{1-e^{-\gamma t}}|g\rangle|\overline{1}\rangle$ and $|\bar{g}\rangle=|g\rangle|\overline{0}\rangle$. The variation of the state $|\bar{e}\rangle$ with time does not produce any the phase to the state $|e\rangle|\overline{0}\rangle$ and has no effect on the acquired geometric phase $\beta$, which is determined by the evolution of the relative probability amplitude of the two joint states $|\bar{e}\rangle$ and $|\bar{g}\rangle$. This allow us to illustrate the geometric aspect of the joint state evolution with the Bloch sphere, whose north pole represents the single-excitation state (the probability of the excitation being held by the atom is irrelevant). With this representation, the coherent evolution corresponds to a rotation of the Bloch vector around the $\mathrm{z}$ axis, and after a complete cycle the system acquires a geometric phase equal to the solid angel subtended by the path followed by vector always pointing to $(\theta, \varphi)$ on the Bloch sphere. Here the system-reservoir entanglement protects the coherence between the single- and zero-excitation states and hence the geometric phase against the energy leakage. 
It is interesting to further illustrate the difference between the present result and that obtained through the quantum jump method with this typical system. According to the quantum jump approach, the geometric phase associated with the no-jump trajectory tends to $\beta-\gamma(\pi \sin \theta)^{2} /(2 B)$ up to the first order in $\gamma / B$. As pointed out in [27], when the environment is monitored and no decaying is observed, the probability of the lower state increases with time so that the state will spiral towards the south pole in the Bloch sphere, changing the area enclosed by the evolution trajectory. This leads to the first order correction. Furthermore, according to the quantum jump approach, the total phase has a dynamical contribution even for $\theta=\pi / 2$. These results are obvious in contrast with the present study, in which the geometric phase depends upon the ratio of the probabilities of the joint states with one and zero excitations which remains unchanged during the system-environment coupling. The geometric phase calculated through the kinematic method [26] also contains a first order correction due to the loss of the system-reservoir entanglement after tracing over the reservoir.

Now we turn to another model in which a two-level atom is driven by a quantized field and coupled to a reservoir. In the frame rotating at the atomic transition frequency, the system Hamiltonian is described by the JC model

$$
H_{s}=\delta a^{\dagger} a+g\left(a^{\dagger}|g\rangle\langle e|+a| e\rangle\langle g|\right)
$$

where $a^{\dagger}$ and $a$ are the creation and annihilation operators for the quantized field, $g$ is the atom-field coupling constant, and $\delta$ is the detuning. Suppose that the quantum system is initially in the state $\left|\psi_{0}^{s}(0)\right\rangle=|e\rangle|0\rangle$, where $|0\rangle$ is the vacuum state of the quantum field. After an interaction time $t$ the system+reservoir state can be written in the form of Eq. (5), where

$$
\begin{aligned}
\left|\psi_{0}^{s}(t)\right\rangle=\frac{1}{2 \lambda} & e^{-(i \delta / 2+\gamma / 4) t}\left\{\left[\left(\lambda+\frac{\delta}{2}+i \frac{\gamma}{4}\right) e^{i \lambda t}\right.\right. \\
& \left.+\left(\lambda-\frac{\delta}{2}-i \frac{\gamma}{4}\right) e^{-i \lambda t}\right]|e\rangle|0\rangle \\
& \left.-g\left(e^{i \lambda t}-e^{-i \lambda t}\right)|g\rangle|1\rangle\right\},
\end{aligned}
$$

with $\lambda=\sqrt{g^{2}+(\delta / 2+i \gamma / 4)^{2}}$. Then the geometric phase of the total system is

$$
\beta=-\delta t / 2+\arg \left\{\frac{1}{2 \lambda}\left[\left(\lambda+\frac{\delta}{2}+i \frac{\gamma}{4}\right) e^{i \lambda t}+\left(\lambda-\frac{\delta}{2}-i \frac{\gamma}{4}\right) e^{-i \lambda t}\right]\right\} .
$$

In the limit $\Omega \gg \gamma$, where $\Omega=\sqrt{g^{2}+\delta^{2} / 4}$, we can expand $\lambda$ up to the second order in $\gamma / \Omega$ : $\lambda \simeq \Omega+i \frac{\delta}{8 \Omega} \gamma-$ $g^{2} \gamma^{2} /\left(32 \Omega^{4}\right)$. After a full Rabi cycle with $T=\pi / \Omega$, the geometric phase is $\beta \simeq \beta^{0}+\pi \delta\left(3 g^{2}-\delta^{2} / 2\right) \gamma^{2} /\left(64 \Omega^{5}\right)$, where $\beta^{0}=\pi[1-\delta /(2 \Omega)]$ is the geometric phase without decoherence. This implies that the geometric phase is equal to $\beta^{0}$ up to the first order in $\gamma / \Omega$, reflecting its robustness against dissipation. Based on previous approaches, the lowest order correction for this open system is linear [37], which is in distinct contrast with the present result. It should be noted that for this open system the geometric phase calculated through the present approach is the only one that can be directly measured in experiment [37].

This result can be understood in the following way. We can rewrite the total system-reservoir Hamiltonian in the single-excitation subspace as

$$
\begin{aligned}
H= & \Omega(|+\rangle\langle+|-|-\rangle\langle-|)+\sum_{k=1}^{N} \omega_{k} b_{k}^{\dagger} b_{k} \\
& +\sum_{k=1}^{N}\left\{g_{k} b_{k}[\cos (\theta / 2)|+\rangle+\sin (\theta / 2)|-\rangle]\langle g, 0|+H . c .\right\},
\end{aligned}
$$

where $|+\rangle=\cos (\theta / 2)|e, 0\rangle+\sin (\theta / 2)|g, 1\rangle$ and $|-\rangle=\sin (\theta / 2)|e, 0\rangle-\cos (\theta / 2)|g, 1\rangle$ are the dressed states of the Jaynes-Cummings system, where $\cos \theta=\delta /(2 \Omega)$. Under the condition that the Rabi frequency $\Omega$ is much larger than the atomic decay rate $\gamma$ (the linewidth of the upper level), the probability for the system undergoing transition from one dressed state to the other vanishes due to the high frequency evolution. In other words, the dressed state $|+\rangle$ $(|-\rangle)$ can only emit a photon into the reservoir modes with the frequency ranging from $\omega_{a}+\Omega-\gamma$ to $\omega_{a}+\Omega+\gamma$ $\left(\omega_{a}-\Omega-\gamma\right.$ to $\left.\omega_{a}-\Omega+\gamma\right)$. When $\gamma \ll \Omega$, the spectra of these two dressed states do not overlap, and they evolve independently in the reservoir, with the corresponding decaying rates $\gamma_{+}=\gamma \cos ^{2}(\theta / 2)$ and $\gamma_{-}=\gamma \sin ^{2}(\theta / 2)$. Then the state evolution of the whole system can be approximately written as

$$
\left|\psi^{s r}(t)\right\rangle \simeq e^{-i(\delta t+\varphi) / 2}\left[\cos (\theta / 2)\left|e_{+}\right\rangle+e^{i \varphi} \sin (\theta / 2)\left|e_{-}\right\rangle\right],
$$


where $\left|e_{ \pm}\right\rangle=e^{-\gamma_{ \pm} t / 2}| \pm\rangle|\overline{0}\rangle+\sqrt{1-e^{-\gamma_{ \pm} t}}|g, 0\rangle\left|\overline{1}_{ \pm}\right\rangle, \varphi=2 \Omega t$, and $\left|\overline{1}_{ \pm}\right\rangle=\sum_{k=1}^{N} \lambda_{k, \pm}\left|0_{1} \ldots 0_{k-1} 1_{k} 0_{k+1} \ldots 0_{N}\right\rangle$ are the normalized collective reservoir states with $\left\langle\overline{1}_{+} \mid \overline{1}_{-}\right\rangle=0$. Similar to the previous case, the time evolution of either of the states $\left|e_{+}\right\rangle$and $\left|e_{-}\right\rangle$has no effect on the acquired geometric phase $\beta$. Therefore, after a time $T=\pi / \Omega$ the acquired geometric phase $\beta$ is also directly related to the solid angle swept by the vector pointing $(\theta, \varphi)$ on the Bloch sphere.

The approach can be generalized to the case when the system is composed of different subsystems, each coupled to an independent reservoir. As an example, we consider the dissipative JC model in which the atomic spontaneous emission and photon decay rates are $\gamma$ and $\kappa$, respectively. Suppose that the system is initially in the state $|e\rangle|n\rangle$. Then the evolution of the state component with no atomic spontaneous decay and photon loss is

$$
\begin{aligned}
\left|\psi_{0}^{s}(t)\right\rangle= & \frac{1}{2 \lambda_{n}} e^{-[(i \delta+\kappa / 2)(2 n+1) / 2+\gamma / 4] t}\left\{\left[\left(\lambda_{n}+\frac{\delta}{2}+i \frac{\gamma-\kappa}{4}\right) e^{i \lambda_{n} t}\right.\right. \\
& \left.+\left(\lambda_{n}-\frac{\delta}{2}-i \frac{\gamma-\kappa}{4}\right) e^{-i \lambda_{n} t}\right]|e\rangle|n\rangle \\
& \left.-g \sqrt{n+1}\left(e^{i \lambda_{n} t}-e^{-i \lambda_{n} t}\right)|g\rangle|n+1\rangle\right\}
\end{aligned}
$$

where $\lambda_{n}=\sqrt{g^{2}(n+1)+[\delta / 2+i(\gamma-\kappa) / 4]^{2}}$. When $(\gamma-\kappa) / \Omega_{n} \ll 1$ with $\Omega_{n}=\sqrt{g^{2}(n+1)+\delta^{2} / 4}$, up to the second order in $(\gamma-\kappa) / \Omega_{n}$ we have $\lambda_{n} \simeq \Omega_{n}+i \frac{\delta}{8 \Omega_{n}}(\gamma-\kappa)-g^{2}(\gamma-\kappa)^{2} /\left(32 \Omega^{4}\right)$. After a time $T=\pi / \Omega_{n}$, the acquired geometric phase is $\beta_{n} \simeq \beta_{n}^{0}+\pi \delta\left(3 g^{2}-\delta^{2} / 2\right)(\gamma-\kappa)^{2} /\left(64 \Omega^{5}\right)$, where $\beta_{n}^{0}=\pi\left[1-\delta /\left(2 \Omega_{n}\right)\right]$ is the result without decoherence. This implies that to the first order correction $\beta_{n}$ is affected neither by the atomic spontaneous decay nor by the photon loss. This phase can also be directly measured in the Ramsey interference experiment by employing an auxiliary state $|f\rangle$ that is not coupled to the quantized field [37].

In conclusion, we have described a new method for calculating the geometric phases of a quantum system subjected to decoherence based on joint system-reservoir evolution. This method requires neither to measure the quantum jumps nor to trace over the reservoir modes, offering a way to get around the nonunitary evolution obstacle. The geometric phase is associated with the curvature of the total system-reservoir Hilbert space and always can be well defined. In particular, we have shown that the geometric phase can be calculated without completely knowing the state evolution of the overall system. Using this method, we have calculated the geometric phase of a qubit evolving under different Hamiltonians and subject to decoherence, and show that for the dispersive system Hamiltonian it is completely insensitive to decoherence. In case of low docoherence, the correction of this phase associated with the JC evolution vanishes up to the first order correction. These results are completely different from previous works, which showed that for nonadiabatic evolution the decoherence effect appears in the first order correction. We have presented a geometric explanation to this difference. Furthermore, we have shown that for the dissipative JC model the geometric phase calculated through the present method is the only one that can be directly detected in experiment. Our method is generic and can be applied to various physical systems subject to spontaneous decay.

This work was supported by the National Natural Science Foundation of China under Grant No. 11374054 and the Major State Basic Research Development Program of China under Grant No. 2012CB921601.

[1] M. V. Berry, Proc. R. Soc. Lond. A 392, 45 (1984).

[2] J. Anandan, Nature 360, 307 (1992).

[3] A. Shapere and F. Wilczek, Geometric Phases in Physics (World Scientific, Singapore, 1989).

[4] Y. Aharonov and J. Anandan, Phys. Rev. Lett. 58, 1593 (1987).

[5] S. Pancharatnam, Proc. Indian Acad. Sci. Sect. A 44, 247 (1956).

[6] J. Samuel and R. Bhandari, Phys. Rev. Lett. 60, 2339 (1988).

[7] A. Uhlmann, Rep. Math. Phys. 24, 229 (1986).

[8] E. Sjöqvist et al., Phys. Rev. Lett. 85, 2845 (2000).

[9] P. Zanardi and M. Rasetti, Phys. Lett. A 264, 94 (1999).

[10] J.A. Jones, V. Vedral, A. Ekert, and G. Castagnoli, Nature 403, 869 (2000).

[11] G. Falci, R. Fazio, G.M. Palma, J. Siewert, and V. Vedral, Naute 407, 355 (2000).

[12] L.M. Duan, J.I. Cirac, and P. Zoller, Science 292, 1695 (2001).

[13] X.B. Wang and M. Keiji, Phys. Rev. Lett. 87, 097901 (2001).

[14] S.L. Zhu and Z. D. Wang, Phys. Rev. Lett. 89, 097902 (2002).

[15] D. Leibfried et al., Nature 422, 412 (2003). 
[16] S.B. Zheng, Phys. Rev. A 70, 052320 (2004).

[17] G. De Chiara and G. M. Palma, Phys. Rev. Lett. 91, 090404 (2003).

[18] A. Nazir, T. Spiller, and W. J. Munro, Phys. Rev. A 65, 042303 (2002).

[19] A. Blais and A.-M. S. Tremblay, Phys. Rev. A 67, 012308 (2003).

[20] P. Solinas, M. Sassetti, P. Truini, and N. Zangh , New J. Phys. 14, 093006 (2012).

[21] P. J. Leek et al., Science 318, 1889 (2007).

[22] S. Filipp, J. Klepp, Y. Hasegawa, C. Plonka-Spehr, U. Schmidt, P. Geltenbort, and H. Rauch, Phys. Rev. Lett. 102, 030404 (2009).

[23] S. Berger, M. Pechal, A. A. Abdumalikov Jr., C. Eichler, L. Steffen, A. Fedorov, A. Wallraff, S. Filipp, Phys. Rev. A 87, 060303(R) (2013).

[24] S.B. Zheng, Phys. Rev. A 85, 022128 (2012).

[25] M. Ericsson, E. Sjöqvist, J. Brännlund, D. K. L. Oi, and A. K. Pati, Phys. Rev. A 67, 020101(R) (2003).

[26] D. M. Tong, E. Sjoqvist, L. C. Kwek, and C. H. Oh, Phys. Rev. Lett. 93, 080405 (2004).

[27] A. Carollo, I. Fuentes-Guridi, M. F. Santos, and V. Vedral, Phys. Rev. Lett. 90, 160402 (2003).

[28] A. Carollo, I. Fuentes-Guridi, M. F. Santos, and V. Vedral, Phys. Rev. Lett. 92, 020402 (2004).

[29] R. S. Whitney and Y. Gefen, Phys. Rev. Lett. 90, 190402 (2003).

[30] R. S. Whitney, Y. Makhlin, A. Shnirman, and Y. Gefen, Phys. Rev. Lett. 94, 070407 (2005).

[31] X.X. Yi, D. M. Tong, L.C. Wang, L.C. Kwek, and C.H. Oh, Phys. Rev. A 73, 052103 (2006)

[32] P. Solinas, M. Mottonen, J. Salmilehto, and J. P. Pekola, Phys. Rev. B 82, 134517 (2010).

[33] F.C. Lombardo, P.I. Villar, Phys. Rev. A 81, 022115 (2010).

[34] P.I. Villar, and F.C. Lombardo, Phys. Rev. A 83, 052121 (2011).

[35] F.M. Cucchietti, J.-F. Zhang, F.C. Lombardo, P.I. Villar, R. Laflamme, Phys. Rev. Lett. 105, 240406 (2010).

[36] M. Ericsson, A.K. Pati, E. Sjöqvist1, J. Brännlund, and D.K.L. Oi, Phys. Rev. Lett. 91, 090405 (2003).

[37] see supplementary material.

[38] M.O. Scully and M.S. Zubairy, Quantum Optics (Cambridge University Press, Cambridge, 1997).

\section{SUPPLEMENTARY INFORMATION: OPEN SYSTEM GEOMETRIC PHASE BASED ON SYSTEM-RESERVOIR JOINT STATE EVOLUTION}

In the supplementary material we show how to measure the open system geometric phase calculated through our approach in experiment.

We here show how the geometric phase associated with the joint system-reservoir state evolution can be measured in interference experiments. When the system is initially in the state $\left|\psi_{0}^{s}(0)\right\rangle$, after a cyclic evolution the system-reservoir joint state can be written as

$$
\eta e^{i \phi}\left|\psi_{0}^{s}(0)\right\rangle|\overline{0}\rangle+\sqrt{1-\eta^{2}}\left|\psi^{\prime} s r\right\rangle
$$

where $\left|\psi^{\prime} s r\right\rangle$ is the joint state component that is orthogonal to $\left|\psi_{0}^{s}(0)\right\rangle|\overline{0}\rangle, \eta$ is a positive and real number, and $\phi$ is the total phase obtained by the system+reservoir state. To measure this phase, we should use a system reference state $|a\rangle$ that does not evolve. Suppose the system is initially prepared in the superposition state $\left[\left|\psi_{0}^{s}(0)\right\rangle+|a\rangle\right] / \sqrt{2}$. After the cyclic evolution, the system+reservoir state is

$$
\frac{1}{\sqrt{2}}\left\{\left[\eta e^{i \phi}\left|\psi_{0}^{s}(0)\right\rangle+|a\rangle\right]|\overline{0}\rangle+\sqrt{1-\eta^{2}}\left|\psi^{\prime} s r\right\rangle\right\}
$$

By this way the total phase $\phi$ is encoded in the relative probability amplitude associated with the states $\left|\psi_{0}^{s}(0)\right\rangle$ and $|a\rangle$. When the dynamical phase is zero, the geometric phase $\beta$ can be directly measured by the relevant interference experiment.

Let us illustrate the procedure for measuring this geometric phase with a typical example. Consider the dissipative Jaynes-Cummings model in which a two-level atom is driven by a quantized field and coupled to a reservoir. The system Hamiltonian $H_{s}$ for this model is given by Eq. (10) of the main text. We aim to measure the geometric phase associated with the evolution of the initial state $\left|\psi_{0}^{s}(0)\right\rangle|\overline{0}\rangle=|e, 0\rangle|\overline{0}\rangle$. Since $\left\langle\psi_{0}^{s}(0)\left|H_{s}\right| \psi_{0}^{s}(0)\right\rangle=0$, no dynamical phase is accumulated during the evolution. The ground state $|g, 0\rangle$ does not evolve and hence can be used as the reference state. The system is initially prepared in the state $(|e\rangle+|g\rangle)|0\rangle / \sqrt{2}$. In the limit $\Omega=\sqrt{g^{2}+\delta^{2} / 4} \gg \gamma$, 
after a Rabi cycle $(T=\pi / \Omega)$ the system-reservoir state approximately evolves to

$$
\begin{aligned}
\left|\psi^{s r}(T)\right\rangle \simeq & \frac{1}{\sqrt{2}} e^{i \beta}(u|e, 0\rangle+v|g, 1\rangle)|\overline{0}\rangle+ \\
& +\xi|g, 0\rangle|\overline{1}\rangle+\frac{1}{\sqrt{2}}|g, 0\rangle|\overline{0}\rangle
\end{aligned}
$$

where $u=1-\frac{1}{4} \gamma T\left(1+\cos ^{2} \theta\right), v=\frac{1}{8} \gamma T \sin (2 \theta), \xi=\frac{1}{\sqrt{2}} \sqrt{1-u^{2}-v^{2}}, \cos \theta=\delta /(2 \Omega)$. Then we perform the transformation on the atom: $|g\rangle \rightarrow(|g\rangle-|e\rangle) / \sqrt{2}$ and $|e\rangle \rightarrow(|e\rangle+|g\rangle) / \sqrt{2}$, leading to

$$
\begin{aligned}
\left|\psi^{\prime}{ }^{s r}(T)\right\rangle \simeq & \frac{1}{2}\left[\left(1+u e^{i \beta}\right)|g\rangle+\left(u e^{i \beta}-1\right)|e\rangle\right]|0\rangle|\overline{0}\rangle \\
& +\frac{1}{\sqrt{2}}(|g\rangle-|e\rangle)\left(\frac{1}{\sqrt{2}} v e^{i \beta}|1\rangle|\overline{0}\rangle+\xi|0\rangle|\overline{1}\rangle\right) .
\end{aligned}
$$

The final probability for detecting the atom in the state $|g\rangle$ is $P_{g}=\frac{1}{2}(1+u \cos \beta)$. Therefore, we have $\cos \beta=$ $\left(2 P_{g}-1\right) / u$, which implies that the geometric phase based on our approach can be directly measured through the Ramsey interference experiment [1]. Here we have assumed that there is only one decaying channel for the excited state $|e\rangle$. In the presence of more than one lower states and hence decaying channels, the geometric phase remains unchanged, while the probability $P_{g}$ becomes $P_{g}=\frac{1}{4}\left[\left(1+\gamma_{g} / \gamma\right)+\left(u^{2}+v^{2}\right)\left(1-\gamma_{g} / \gamma\right)\right]+\frac{1}{2} u \cos \beta$, where $\gamma_{g}$ is the spontaneous emission rate for decaying to $|g\rangle$. It should be noted that according to the previous approaches $[2,3]$ the total phase for this open system contains a dynamical contribution $\phi_{d} \simeq-\pi^{2} g^{2} \delta \gamma /\left(8 \Omega^{4}\right)$, and hence the corresponding geometric phase cannot be directly measured in experiment. In other words, with the previous definitions the phase measured in the Ramsey interference contains a dynamical component. Based on these method, the correction of the geometric phase due to decoherence is $\pi^{2} g^{2} \delta \gamma /\left(8 \Omega^{4}\right)$ up to the first order in $\gamma / \Omega$.

For the case that the quantized field initially contains $n$ photons and the cavity dissipation is also considered, we should use an auxiliary state $|f\rangle$ that is not coupled to the quantized field to measure the acquired geometric phase. The states $|g\rangle$ and $|f\rangle$ can be chosen to be two hyperfine levels of the atomic ground state. The system is initially prepared in the state $(|e\rangle+|f\rangle)|n\rangle / \sqrt{2}$. Under the condition $\Omega_{n}=\sqrt{g^{2}(n+1)+\delta^{2} / 4} \gg \gamma,(n+1) \kappa$, after a Rabi cycle $\left(T=\pi / \Omega_{n}\right)$ the system-reservoir state approximates

$$
\begin{aligned}
\left|\psi^{s r}(T)\right\rangle \simeq & \frac{1}{\sqrt{2}} e^{i \beta_{n}}\left(u_{n}|e, n\rangle+v_{n}|g, n+1\rangle\right)|\overline{0}\rangle_{a}|\overline{0}\rangle_{p}+ \\
& +\xi_{n}\left[\frac{p_{n}}{\sqrt{2}}\left(|g, n\rangle\left|\overline{1}_{g}\right\rangle_{a}+|f, n\rangle\left|\overline{1_{f}}\right\rangle_{a}\right)|\overline{0}\rangle_{p}\right. \\
& \left.+\left(q_{n}|e, n-1\rangle+s_{n}|g, n\rangle\right)|\overline{0}\rangle_{a}|\overline{1}\rangle_{p}\right]+\frac{1}{\sqrt{2}} e^{-n \kappa T / 2}|f, n\rangle|\overline{0}\rangle_{a}|\overline{0}\rangle_{p} \\
& +\frac{1}{\sqrt{2}} \sqrt{1-e^{-n \kappa T}}|f, n-1\rangle|\overline{0}\rangle_{a}|\overline{1}\rangle_{p},
\end{aligned}
$$

where $u_{n}=1-\frac{1}{4}\left[(2 n+1) \kappa+\gamma+(\gamma-\kappa) \cos ^{2} \theta_{n}\right] T, v_{n}=\frac{1}{8}(\gamma-\kappa) T \sin \left(2 \theta_{n}\right), \xi_{n}=\frac{1}{\sqrt{2}} \sqrt{1-u_{n}^{2}-v_{n}^{2}}, p_{n}=$ $\sqrt{\frac{\gamma\left(1+\cos ^{2} \theta_{n}\right)}{\gamma\left(1+\cos ^{2} \theta_{n}\right)+\kappa\left(2 n+\sin ^{2} \theta_{n}\right)}}, q_{n}=\sqrt{\frac{n \kappa\left(1+\cos ^{2} \theta_{n}\right)}{\gamma\left(1+\cos ^{2} \theta_{n}\right)+\kappa\left(2 n+\sin ^{2} \theta_{n}\right)}}, s_{n}=\sqrt{\frac{(n+1) \kappa \sin ^{2} \theta_{n}}{\gamma\left(1+\cos ^{2} \theta_{n}\right)+\kappa\left(2 n+\sin ^{2} \theta_{n}\right)}}, \cos \theta_{n}=\delta /\left(2 \Omega_{n}\right)$, the subscripts $a$ and $p$ label the reservoirs to which the atom and quantized photonic field are coupled, respectively, and $\left|\overline{1}_{j}\right\rangle_{a}(j=g, f)$ denotes the state of reservoir of the atom associated with decaying to $|j\rangle$. We here have discarded the trivial common phase factor $e^{-i n \delta T}$ and assumed that the spontaneous emission rates for decaying to $|g\rangle$ and $|f\rangle$ 
are both $\gamma / 2$. The subsequent transformations $|f\rangle \rightarrow(|f\rangle-|e\rangle) / \sqrt{2}$ and $|e\rangle \rightarrow(|e\rangle+|f\rangle) / \sqrt{2}$ leading to

$$
\begin{aligned}
\left|\psi^{\prime} s r(T)\right\rangle \simeq \frac{1}{2} & {\left[\left(e^{-n \kappa T / 2}+e^{i \beta_{n}} u_{n}\right)|f, n\rangle+\left(e^{i \beta_{n}} u_{n}-e^{-n \kappa T / 2}\right)|e, n\rangle\right.} \\
+ & \left.\sqrt{2} v_{n}|g, n+1\rangle\right]|\overline{0}\rangle_{a}|\overline{0}\rangle_{p} \\
& +\xi_{n}\left\{\frac{p_{n}}{\sqrt{2}}\left[|g, n\rangle\left|\overline{1}_{g}\right\rangle_{a}+\frac{1}{\sqrt{2}}(|f\rangle-|e\rangle)|n\rangle\left|\overline{1}_{f}\right\rangle_{a}\right]|\overline{0}\rangle_{p}\right. \\
+ & {\left.\left[\frac{1}{\sqrt{2}} q_{n}(|e\rangle+|f\rangle)|n-1\rangle+s_{n}|g, n\rangle\right]|-\rangle_{a}|\overline{1}\rangle_{p}\right\} } \\
+ & \frac{1}{2} \sqrt{1-e^{-n \kappa T}}(|f\rangle-|e\rangle)|n-1\rangle|\overline{0}\rangle_{a}|\overline{1}\rangle_{p} .
\end{aligned}
$$

In this case the final probability for detecting the atom in the state $|f\rangle$ is directly related to the geometric phase: $P_{f}=\frac{1}{4}\left[1+u_{n}^{2}+\xi_{n}^{2}\left(p_{n}^{2}+2 q_{n}^{2}\right)\right]+\frac{1}{2} u_{n} e^{-n \kappa T / 2} \cos \beta$. According to the previous approaches $[2,3]$, for this open system the measured phase contains a dynamical component $\phi_{d} \simeq-\pi^{2} g^{2} \delta(n+1)(\gamma-\kappa) /\left(8 \Omega^{4}\right)$.

[1] P. Bertet, S. Osnaghi, A. Rauschenbeutel, G. Nogues, A. Auffeves, M. Brune, J.M. Raimond, and S. Haroche, Nature 411, 166 (2001).

[2] D. M. Tong, E. Sjoqvist, L. C. Kwek, and C. H. Oh, Phys. Rev. Lett. 93, 080405 (2004).

[3] A. Carollo, I. Fuentes-Guridi, M. F. Santos, and V. Vedral, Phys. Rev. Lett. 90, 160402 (2003). 